\title{
TCL1 expression patterns in Waldenström macroglobulinemia
}

Richard Lemal ${ }^{1,2}$, Sandrine Bard-Sorel ${ }^{3}$, Laura Montrieul ${ }^{1,2}$, Jacques-Olivier Bay ${ }^{1,2}$, Aurélie Ravinet ${ }^{1,2}$, Albane Ledoux-Pilon ${ }^{3}$, Nicolas Cagnard ${ }^{4}$, Sébastien Bailly ${ }^{1,2}$, ${\text { Pierre } \text { Morel }^{5} \text {, Frédéric Charlotte }}^{6}$, Xavier Leleu ${ }^{7,8}$, Stéphanie Poulain ${ }^{9,10,11}$, Pierre J Déchelotte ${ }^{2}$, Olivier Hermine ${ }^{12,13,14}$, Véronique Leblond ${ }^{15,16}$, Olivier Tournilhac ${ }^{1,2}$ and Romain Guièze ${ }^{1,2}$

${ }^{1}$ CHU Clermont-Ferrand, Service d'Hématologie Clinique Adulte et de Thérapie Cellulaire, F-63003 ClermontFerrand, France; ${ }^{2}$ Clermont Université, Université d'Auvergne, EA7283, CIC501, BP 10448, F-63000 ClermontFerrand, France; ${ }^{3} \mathrm{CHU}$ Clermont-Ferrand, Service d'Anatomie Pathologique, F-63003 Clermont-Ferrand, France; ${ }^{4}$ Plateforme Bio-informatique Paris Descartes, Institut Necker Enfants Malades (INEM), Paris, France; ${ }^{5}$ Service d'Hématologie, CH, Lens, France; ${ }^{6}$ APHP Hôpital Pitié-Salpêtrière, Anatomie Pathologique, Paris, France; ${ }^{7}$ Service des Maladies du Sang, Hôpital Huriez, CHRU Lille, Lille, France; ${ }^{8}$ U837 INSERM, IRCL, CHRU, Lille, France; ${ }^{9}$ Service d'Hématologie-Immunologie-Cytogénétique, Centre Hospitalier de Valenciennes, France; ${ }^{10}$ Laboratoire d'Hématologie, Centre de Biologie et Pathologie, CHRU Lille, France; ${ }^{11}$ INSERM UMR 1172, IRCL, Lille, France; ${ }^{12}$ Department of Clinical Hematology, Necker Hospital, Paris, France; ${ }^{13}$ INSERM UMR 1163, Laboratory of Cellular and Molecular Mechanisms of Hematological Disorders and Therapeutic Implications, Paris, France; ${ }^{14}$ Paris Descartes Sorbonne Paris Cité University, Imagine Institute, Paris, France; ${ }^{15}$ APHP Hôpital Pitié-Salpêtrière, Hématologie, Paris, France and ${ }^{16}$ UPMC Paris 6 , GRC-11, GRECHY, Paris, France

The oncogenic role of TCL1 in chronic lymphocytic leukemia is well established in transgenic mice. TCL1 expression in other B-cell malignancies has been also described: post-germinal center-derived malignancies, such as multiple myeloma, classically do not express TCL1. Waldenström macroglobulinemia is a post-germinal center malignancy that is known to be similar to chronic lymphocytic leukemia in terms of its gene expression profile. TCL1 expression has not been so far assessed in Waldenström macroglobulinemia. Transcriptomic explorations show that TCL1A expression is linked to signaling pathways and biological functions that are known to be involved in Waldenström macroglobulinemia as well as to gene signatures of interest in B-cell malignancies. We investigated TCL1 expression at the protein level in the bone marrow of a series of 59 patients with Waldenström macroglobulinemia: $76 \%$ of patients expressed TCL1, which appeared to be associated with a pejorative prognostic impact. TCL1 could have an oncogenic role in Waldenström macroglobulinemia, and deserves further exploration.

Modern Pathology (2016) 29, 83-88; doi:10.1038/modpathol.2015.122; published online 23 October 2015

Waldenström macroglobulinemia is an uncommon B-cell lymphoproliferative disorder characterized by lymphoplasmacytic cells that infiltrate the bone marrow, along with the presence of an immunoglobulin $\mathrm{M}$ monoclonal protein in the serum. ${ }^{1}$

Correspondence: Dr R Guièze, MD, CHU Clermont-Ferrand, Service d'Hématologie Clinique Adulte et de Thérapie Cellulaire, EA7283, CIC501, Clermont Université, Université d'Auvergne, CHU Estaing, 1 place Lucie Aubrac, 63003 Clermont-Ferrand, France.

E-mail: rguieze@chu-clermontferrand.fr

Received 16 June 2015; revised 12 September 2015; accepted 13 September 2015; published online 23 October 2015
Waldenström macroglobulinemia is thought to be derived from $\mathrm{B}$ cells in which differentiation has been arrested after a somatic hypermutation process in the germinal center and before terminal maturation into plasma cells. ${ }^{2}$ Oncogenesis may be a result of dysregulation of the NF-kB and PI3K/Akt/ mTOR signaling pathways, ${ }^{3-5}$ and from the influence of the bone marrow microenvironment. ${ }^{6,7}$ Furthermore, whole-genome sequencing of Waldenström macroglobulinemia patients has recently uncovered highly recurring $M Y D 88^{\mathrm{L} 265 \mathrm{P}}$ and WHIM-like $C X C R 4$ mutations that have a potential role in such pathways. ${ }^{8,9}$ 
The T-cell leukemia/lymphoma 1 (TCL1) protein encoded by the TCL1A gene (TCL1) is normally expressed during the early steps of $\mathrm{B}$ - and T-cell lymphopoiesis. ${ }^{10}$ TCL1 has been shown to be overexpressed in chronic lymphocytic leukemia, ${ }^{11,12}$ and its oncogenic role has been well established in transgenic mice. ${ }^{13}$ It acts as an activator of $\mathrm{Akt}^{14}$ and NF-kB ${ }^{15}$ signaling in the cytoplasm, but may also function as a transcriptional regulator in the nucleus. ${ }^{16}$ Increased TCL1 expression has been observed in other B-cell disorders ${ }^{17,18}$ but not in multiple myeloma. TCL1 expression level still needs to be investigated in Waldenström macroglobulinemia and is the focus of this report.

\section{Materials and methods}

\section{Analysis of Transcriptomic Datasets}

Transcriptomic data were obtained from native data taken from two publicly available datasets (GSE6691 and GSE12668 datasets). The GSE6691 GEO dataset (56 samples) provided insight into the differences between cells from Waldenström macroglobulinemia and their cell counterparts in chronic lymphocytic leukemia and multiple myeloma. These data were analyzed to compare respective TCL1A expressions in these disease entities using the GEO2R tool from NCBI. The GSE12668 dataset (22 samples) provided high-resolution genomic analysis of Waldenström macroglobulinemia. These data were analyzed to assess TCL1A-co-regulated genes in Waldenström macroglobulinemia. We performed a new normalization procedure to check the intrinsic qualities of the native data: gene expression levels were normalized using the GC Robust Multi-array Average algorithm and flags were computed using MAS5. Quality assessment of the chips was performed using the affyQC-Report $\mathrm{R}$ package. Cluster analysis was performed by hierarchical clustering using Spearman's correlation of similarity measure and an average-linkage algorithm. Data were analyzed using QIAGEN's Ingenuity Pathway Analysis, ${ }^{19}$ which assessed the relationships between genes and proteins, and constructed pathways to unveil the relevant biological processes.

\section{Patients and Samples}

Fifty-nine patients who underwent a bone marrow trephine biopsy for Waldenström macroglobulinemia, at Clermont-Ferrand and La Pitié-Salpêtrière University Hospitals between 1998 and 2012, were included in accordance with the Declaration of Helsinki. All included patients presented with Waldenström macroglobulinemia as defined by the international guidelines according to the World Health Organization classification ${ }^{20}$ and the Second International Workshop on Waldenström's
Macroglobulinemia, ${ }^{1}$ ie, bone marrow infiltrated by immunoglobulin $M$ producing clonal lymphoplasmacytic lymphoma. All patients were treated according to the Athens criteria. ${ }^{1}$ Their characteristics are shown in Table 1 . The median follow-up was 103 months (6-261 months).

\section{Immunohistochemistry and Scoring}

Analyses were performed in the Clermont-Ferrand pathology laboratory using monoclonal mouse antihuman TCL1 antibody (Clone 27D6/20; MBL International Corporation, Des Plaines, IL, USA; 1/100 dilution for $1 \mathrm{~h}$ ) and monoclonal mouse anti-human CD20 antibody (Clone L26; Dako, Carpinteria, CA, USA; 1/100 dilution for $1 \mathrm{~h}$ ). Immunostaining was assessed by three independent investigators who were blinded to the clinical data. If there was disagreement, a second review was conducted.

To assess the tumor infiltrates (stained by hematoxylin and eosin, and by anti-CD20 antibody), the percentage of TCL1-stained tumor cells was determined using a previously published four-tier scoring system for TCL1 assessment in B-cell malignancies, ${ }^{11,17}$ as follows: $0=$ negative; $1=<5 \%$ of tumor cells positive; $2=$ positive in up to $50 \%$ of tumor cells; and $3=$ positive in $>50 \%$ of tumor cells. TCL1 staining intensity was also recorded using a previously published three-tier score $(0=$ no staining; $1=$ low intensity; $2=$ high intensity) ${ }^{11}$ to evaluate the intra-tumoral distribution and level of TCL1 expression within each tumor sample.

\section{Statistical Analyses}

Therapeutic requirements and the time until endpoints were defined according to published recommendations. ${ }^{1,21,22}$ Pearson Chi-square test, two-sided Fisher test, Mann-Whitney test, Kaplan-Meier method, and log-rank test were applied to the data in appropriate settings, using SPSS Statistics v22 (IBM), PRISM v6.0 (Graphpad), and/or $\mathrm{R}^{23}$ software.

\section{Results}

\section{TCL1A Expression and Related Transcriptome}

To investigate the potential role of TCL1 in the biology of Waldenström macroglobulinemia, we first analyzed publicly available transcriptomic datasets. Analyses of data from GSE6691 showed that TCL1A mRNA expression levels of Waldenström macroglobulinemia cells ranged from those observed in multiple myeloma to those observed in chronic lymphocytic leukemia $(P<0.001)$ (Figure 1a). In order to analyze TCL1-related transcriptome, we defined two groups of patients according to TCL1A expression using the GSE12668 dataset: ie, a TCL1A ${ }^{\text {low }}$ group with normalized fluorescence of 
Table 1 Patients' characteristics

\begin{tabular}{|c|c|c|c|c|}
\hline & $\begin{array}{c}A L L \\
(\mathrm{n}=59)\end{array}$ & $\begin{array}{c}\text { Neg./weak TCL1A } \\
(\mathrm{n}=16)\end{array}$ & $\begin{array}{c}\text { Mod./strong TCL1A } \\
(\mathrm{n}=43)\end{array}$ & $\mathrm{P}$ \\
\hline Sex ratio $(\mathrm{M} / \mathrm{F})$ & $37 / 22$ & $12 / 4$ & $25 / 18$ & 0.23 \\
\hline Age at diagnosis, median (range), years & $60(36-83)$ & $64(36-83)$ & $59(37-80)$ & 0.15 \\
\hline \multicolumn{5}{|l|}{$I S S-W M(\mathrm{n}=54)$} \\
\hline Low risk, $N(\%)$ & $14(26)$ & $4(29)$ & $10(25)$ & 1 \\
\hline Int/High risk, $N(\%)$ & $40(74)$ & $10(71)$ & $30(75)$ & \\
\hline \multicolumn{5}{|l|}{ Biology } \\
\hline $\mathrm{Hb}$, median (range), g/dl & $10.5(5.4-16.5)$ & $9.8(5.4-14.5)$ & $10.6(5.6-16.5)$ & 0.24 \\
\hline Hb level $\leq 11.5 \mathrm{~g} / \mathrm{dl}, N(\%)$ & $41(69)$ & $12(75)$ & $29(67)$ & 0.75 \\
\hline Platelet count, median (range), $\times 10^{9} / 1$ & $213(18-430)$ & $190(37-422)$ & $224(18-430)$ & 0.25 \\
\hline Platelet count $<100 \times 10^{9} / 1, N(\%)$ & $4(7)$ & $3(19)$ & $1(2)$ & 0.057 \\
\hline$\beta 2$ microglobulin, median (range), mg/l $(n=40)$ & $3.3(1.4-9.8)$ & $2.9(2.1-9.8)$ & $3.5(1.4-6.3)$ & 0.73 \\
\hline$\beta 2$ microglobulin $>3 \mathrm{mg} / \mathrm{l}, N(\%)(n=46)$ & $27(46)$ & $4(36)$ & $23(66)$ & 0.16 \\
\hline Monoclonal component, median (range), g/l & $26.2(2.3-87)$ & $33.1(8.3-55.2)$ & $25.5(2.3-87)$ & 0.54 \\
\hline Monoclonal component $>70 \mathrm{~g} / \mathrm{l}, N(\%)$ & $2(3)$ & 0 & $2(5)$ & 1 \\
\hline Lymphocytosis, median (range), $\times 10^{9} / 1$ & $1.5(0.42-6.1)$ & $1.5(0.54-6.1)$ & $1.74(0.42-4.9)$ & 0.5 \\
\hline Absolute neutrophil count, median (range) $\times 10^{9} / 1$ & $3(0.06-10)$ & $3.7(0.06-10)$ & $2.7(1.7-9.6)$ & 0.064 \\
\hline $\mathrm{LDH}>$ Normal, $N(\%)(n=52)$ & $6(10)$ & $1(8)$ & $5(13)$ & 1 \\
\hline \multicolumn{5}{|l|}{ Clinical features, N (\%) } \\
\hline Hyperviscosity & $10(17)$ & $3(19)$ & $7(13)$ & 1 \\
\hline Adenopathy & $27(46)$ & $9(56)$ & $18(42)$ & 0.32 \\
\hline Splenomegaly & $13(22)$ & $1(6)$ & $12(28)$ & 0.09 \\
\hline Hepatomegaly & $11(19)$ & $1(6)$ & $10(23)$ & 0.26 \\
\hline Neuropathy & $11(19)$ & $4(25)$ & $7(16)$ & 0.47 \\
\hline \multicolumn{5}{|l|}{ Treatment, N (\%) } \\
\hline Rituximab & $5(9)$ & $1(6)$ & $4(9)$ & \\
\hline Chlorambucil & $27(46)$ & $5(31)$ & $22(51)$ & \\
\hline Fludarabine & $12(20)$ & $4(25)$ & 8 (19) & \\
\hline Fludarabine, cyclophosphamide & $2(3)$ & 0 & $2(5)$ & \\
\hline Rituximab, fludarabine, cyclophosphamide & $3(5)$ & $2(13)$ & $1(2)$ & \\
\hline Dexamethasone, rituximab, cyclophosphamide & $9(15)$ & $4(25)$ & $5(12)$ & \\
\hline Bendamustine, rituximab & $1(2)$ & 0 & $1(2)$ & \\
\hline
\end{tabular}

285 ( $<25$ th percentile) and a TCL1 $A^{\text {high }}$ group with normalized fluorescence of 3186 ( $>75^{\text {th }}$ percentile).

We checked that median TCL1A expression in the TCL1 $A^{\text {low }}$ group was 10-fold higher than the background noise and that TCL1A expression was significantly different between both groups (mean TCL $1 A^{\text {high }} / T C L 1 A^{\text {low }}$ ratio of 11 , and a mean delta $\left(T C L 1 A^{\text {high }}-T C L 1 A^{\text {low }}\right)$ of 2901$)(P<0.001)$. We then compared the gene expression profile related to each group (TCL1 $A^{\text {high }}$ vs TCL1 $A^{\text {low }}$ ).

Consistent with the oncogenic role of TCL1, most relevant signaling pathways associated with TCL1 $A^{\text {high }}$ expression are known to be involved in cell cycle, apoptosis, and the DNA-damage response. Interestingly, TCL1 $A^{\text {high }}$ expression was associated with signaling pathways previously shown to be relevant in Waldenström macroglobulinemia (ie, PI3K/AKT, mTOR, BCR signal ${ }^{3-5}$ ) (Figure 1b). The highlighted biological functions related to TCL1A agreed with these results (Supplementary Table 1). In addition, TCL1 $A^{\text {high }}$ was associated with gene signatures of interest in tumor B-cell progression or resistance (eg, activated MYC, CCND1, SOX11 signatures, and an inhibited TP53 signature) (Supplementary Table 2).
By computing these data and using published data on TCL1A, we explored the putative interactions of TCL1A in Waldenström macroglobulinemia tumor cells. TCL1A could be linked to crucial actors in the pathophysiology of Waldenström macroglobulinemia within a tightly coupled network (Figure 1c).

\section{Impact of TCL1 Protein Expression on Survival}

Immunostaining of bone marrow tumor cells revealed that TCL1 was expressed at the protein level in 45 (76\%) Waldenström macroglobulinemia patients. This expression was weak in 2 , moderate in 17, and strong in 26 patients (Figure 2a). TCL1 was preferentially detected in the cytoplasm compared with the nucleus.

Regarding the clinical relevance of TCL1 expression in Waldenström macroglobulinemia, we failed to demonstrate any significant correlation between TCL1 expression pattern and the Waldenström macroglobulinemia features known to affect the prognosis (Table 1). However, patients with moderate/strong TCL1 expression had a significantly shorter time to next treatment compared with patients with negative/ weak expression (16.4 vs 59.4 months, respectively; 


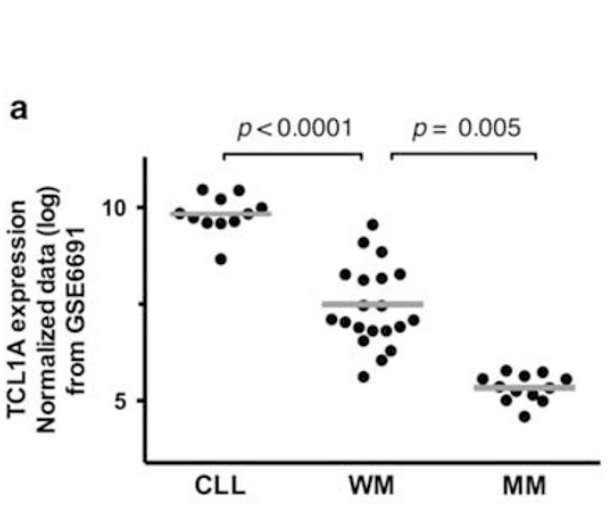

b

\begin{tabular}{|c|c|c|c|}
\hline & Canonical Pathwavs & $-\log (\mathrm{p}$-value $)$ & Ratio \\
\hline \multirow{4}{*}{ Cell cycle } & Cell Cycle: G2/M DNA Damage Checkpoint Regulation & 1.23E01 & 4.49E-01 \\
\hline & Mitotic Roles of Polo-Like Kinase & 1.09E01 & 3.64E-01 \\
\hline & Cell Cycle Control of Chromosomal Replication & $5.9 \mathrm{E} 00$ & 4.07E-01 \\
\hline & Cyclins and Cell Cycle Regulation & $5.66 \mathrm{E} 00$ & 2.44E-01 \\
\hline \multirow{4}{*}{ DDR } & Role of CHK Proteins in Cell Cycle Checkpoint Control & $6.72 \mathrm{E} 00$ & 3.09E-01 \\
\hline & Mismatch Repair in Eukaryotes & 6.4IE00 & $5.62 \mathrm{E}-01$ \\
\hline & DNA damage-induced $14-3-3 \sigma$ Signaling & $5.59 \mathrm{E} 00$ & $4.74 \mathrm{E}-01$ \\
\hline & ATM Signaling & $5.52 \mathrm{E} 00$ & 2.7IE-01 \\
\hline BCR signaling & B Cell Receptor Signaling & $5.45 \mathrm{E} 00$ & $1.76 \mathrm{E}-01$ \\
\hline PI3K/AKT & PI3K Signaling in B Lymphocytes & $5.33 \mathrm{E} 00$ & 1.95E-01 \\
\hline PISNAKT & PI3K/AKT Signaling & $5.13 \mathrm{E} 00$ & 1.95E-01 \\
\hline \multirow{13}{*}{ mTOR and protein synthesis } & mTOR Signaling & $9.27 \mathrm{E} 00$ & 2.13E-01 \\
\hline & EIF2 Signaling & $1.72 \mathrm{E} 01$ & 2.81E-01 \\
\hline & Regulation of elF 4 and p $7056 \mathrm{~K}$ Signaling & $1.16 \mathrm{E} 01$ & $2.6 \mathrm{E}-01$ \\
\hline & p70S6K Signaling & $5.39 \mathrm{E} 00$ & 2.02E-01 \\
\hline & DNA Methylation and Transcriptional Repression & & \\
\hline & Signaling & 6.43E00 & $5,00 \mathrm{E}-01$ \\
\hline & RAN Signaling & 6.11E00 & 5.29E-01 \\
\hline & fMLP Signaling in Neutrophils & $5.62 \mathrm{E} 00$ & 2.13E-01 \\
\hline & Telomere Extension by Telomerase & 5.51E00 & $5.33 \mathrm{E}-01$ \\
\hline & Glucocorticoid Receptor Signaling & $5.25 \mathrm{E} 00$ & 1.53E-01 \\
\hline & Telomerase Signaling & $5.16 \mathrm{E} 00$ & 2.12E-01 \\
\hline & Protein Ubiquitination Pathway & 1.06E01 & 2,00E-01 \\
\hline & Oxidative Phosphorylation & $5,00 \mathrm{E}+00$ & $2.02 \mathrm{E}-01$ \\
\hline
\end{tabular}

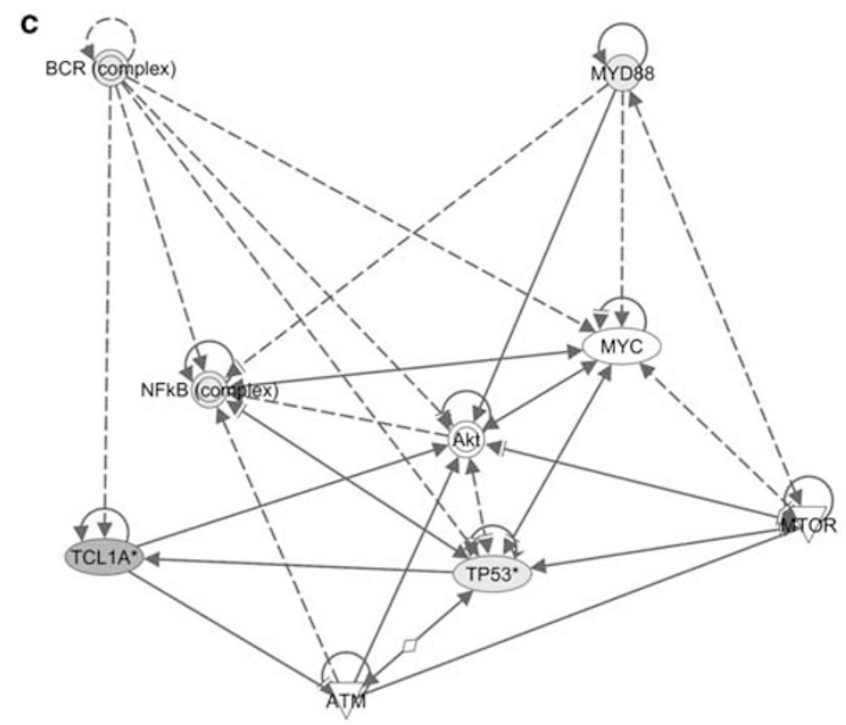

Figure 1 TCL1A transcriptomic expression: comparative exploration and biological relevance. (a) TCL1A transcriptomic expression was compared within three different B-cell malignancies: chronic lymphocytic leukemia (CLL), multiple myeloma (MM), and Waldenström macroglobulinemia (WM). TCL1A transcription level in WM was intermediate between CLL and MM, as was expected regarding the classical precocious post-germinal center normal counterpart in WM. (b) Main canonical pathways were positively linked to a high TCL1A transcription level. In order to show only the strongest and most reliable data, we show all pathways applicable to hematological malignancies that had a $-\log (P$-value $)>5$ and were linked to biological function. It is noteworthy that the main pathways implicated in the pathophysiology of WM are among the canonical pathways linked to TCL1A's high transcription level (BCR, PI3K/Akt, mTOR, and ATM). (c) TCL1A could be directly (full line) or indirectly (dotted line) linked to the main biological actors in WM (actors significantly more expressed in TCL1 $A^{\text {high }}$ cells are colored (red or light grey)). Of note, in this study, MYD88 and TCL1 transcription levels were correlated (MYD88 ratio 1.23; delta: 142; $P=0.024$ ). A full color version of this figure is available at the Modern Pathology journal online.

HR 2.07 (95\% CI: 1.037-3.692), $P=0.036$ ) (Figure 2b). No significant impact was observed in terms of progression-free survival or overall survival.

\section{Discussion}

Herein, for the first time, we have highlighted the expression of the TCL1 oncogene in Waldenström macroglobulinemia. The TCL1 transcriptomic targets were in line with the biology of Waldenström macroglobulinemia, and high TCL1 expression was associated with a poor outcome.
Using analyses from transcriptomic datasets, we found that TCL1 expression levels ranged from those observed in multiple myeloma to those observed in chronic lymphocytic leukemia. Moreover, gene expression profiles related to TCL1 gene expression were similar to the gene signatures of interest in B-cell malignancies, and corresponded to the biological pathways involved in cell cycle, apoptosis, and the DNA-damage response, which are known to be implicated in Waldenström macroglobulinemia oncogenesis. Altogether, these results suggest that TCL1 could have an oncogenic role in Waldenström macroglobulinemia. 

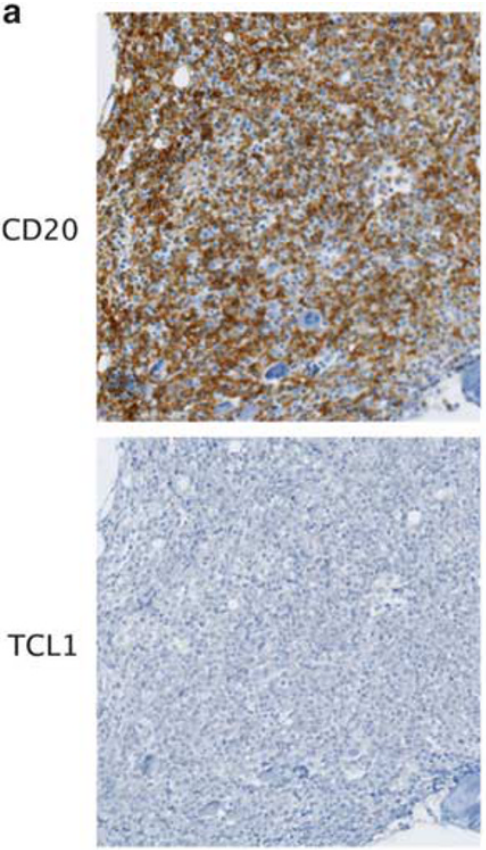

Negative

$\mathrm{n}=\quad 14(24 \%)$
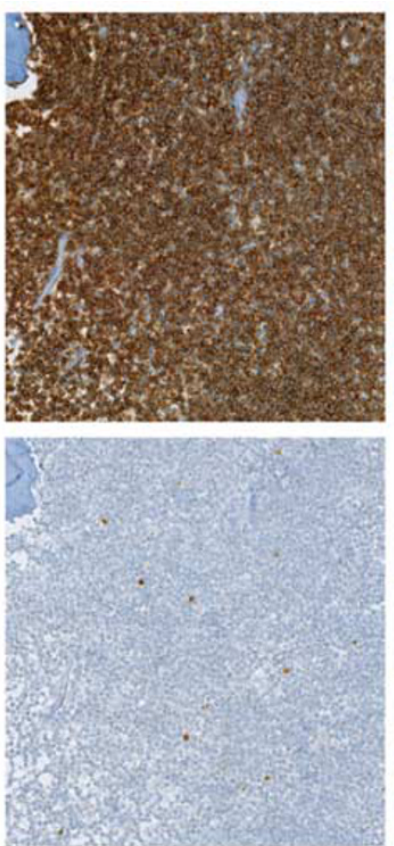

$<5 \%$ of tumor cells

$2(3 \%)$
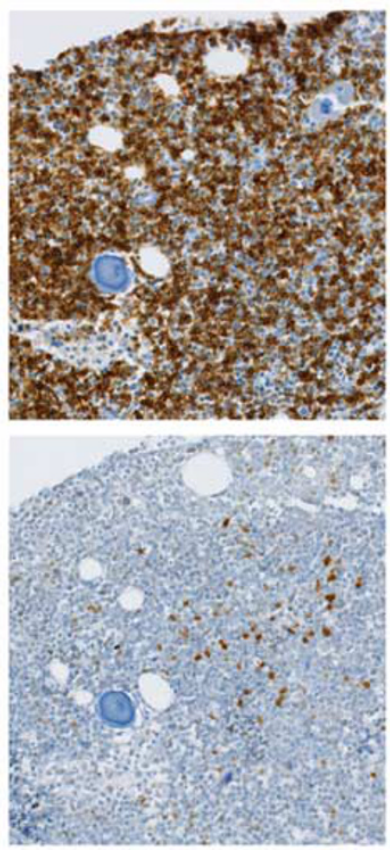

5 to $50 \%$ of tumor cells

$17(29 \%)$
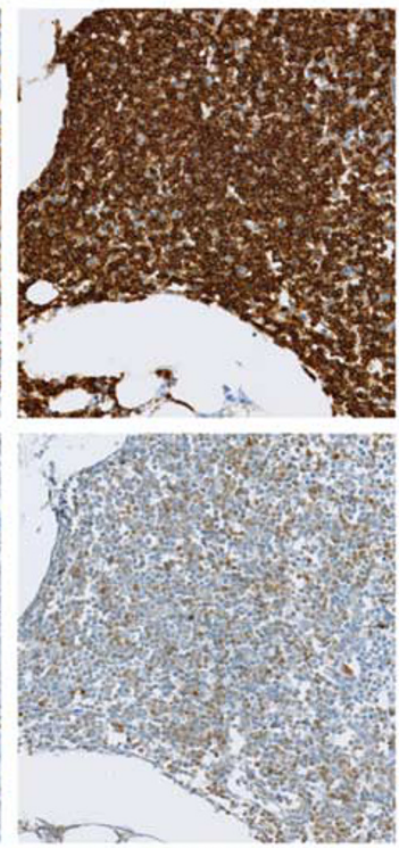

$>50 \%$ of tumor cells

$26(44 \%)$
Negative / Weak TCL1

$16(27 \%)$
Moderate / Strong TCL1

$43(73 \%)$

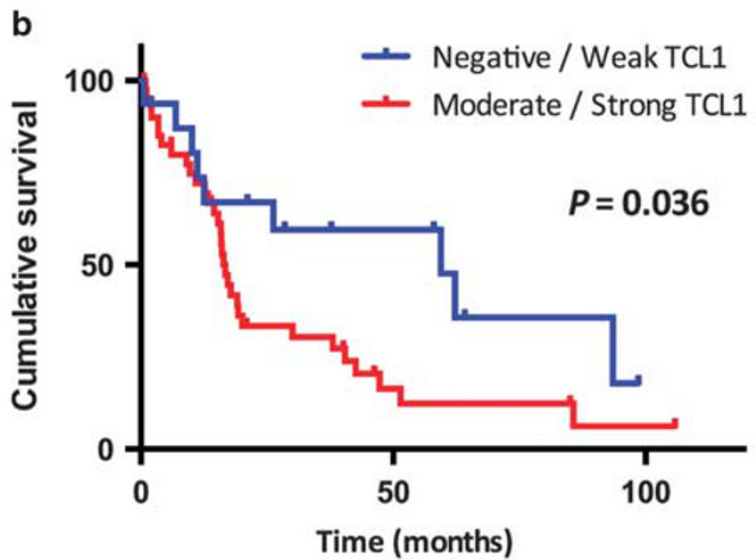

Figure 2 TCL1 protein expression: characteristics and clinical impacts on the time to next treatment. (a) TCL1 protein expression was assessed with a previously published four-tier scoring system that focused on the percentage of CD20+ tumor cells positive for TCL1 staining. Most patients showed 'moderate to strong' expression of TCL1. (b) Patients with moderate/strong TCL1 expression had a shorter time to next treatment compared with those with negative/weak expression (respectively 16.4 vs 59.4 months, HR 2.07 (1.037-3.692), $P=0.036)$.

Most patients expressed TCL1 at a protein level. Moreover, the high percentage of tumor cells that expressed TCL1 was linked to a poor outcome but was not associated with classical agressive features. Interestingly, staining intensity was heterogeneous within the tumor infiltrates, as has been described in chronic lymphocytic leukemia, ${ }^{11}$ which questions the mechanisms underlying TCL1 overexpression in Waldenström macroglobulinemia.

TCL1 was preferentially detected in the cytoplasm compared with the nucleus: this has also been observed in Burkitt's lymphoma, but both cytoplasmic and nuclear localization were then seen in pre-germinal center B-cell lymphoma. ${ }^{17}$

On the basis of these observations, two major issues have to be addressed. First, the published data indicate that TCL1 is not expressed in patients with marginal-zone lymphoma. ${ }^{17,24}$ Differential diagnosis of Waldenström macroglobulinemia with marginalzone lymphoma can be challenging, and its diagnosis directly impacts on the choice of therapeutic options. Our data suggest that TCL1 expression could be a differential marker between Waldenström macroglobulinemia and marginal-zone lymphoma, although 
this remains to be explored further. In addition, if TCL1 expression can clearly distinguish Waldenström macroglobulinemia from other B-cell malignancies, including marginal-zone lymphoma and multiple myeloma, such a difference, as demonstrated by the $M Y D 88^{\mathrm{L} 265 \mathrm{P}}$ mutation, could provide important insights regarding the specific patterns of tumor development in Waldenström macroglobulinemia.

Overall, our results indicate that the TCL1 oncogene is significantly expressed in most of Waldenström macroglobulinemia cases, in which it could drive important pathways and adversely impact on prognosis. Added value of TCL1 staining for Waldenström macroglobulinemia diagnosis remains to be investigated.

\section{Acknowledgments}

We acknowledge Laetitia Pere and Emmanuel Bourgeois from the Clermont-Ferrand pathology laboratory for their technical and logistical assistance.

\section{Disclosure/conflict of interest}

The authors declare no conflict of interests.

\section{References}

1 Owen RG, Treon SP, Al-Katib A et al. Clinicopathological definition of Waldenstrom's macroglobulinemia: consensus panel recommendations from the Second International Workshop on Waldenstrom's Macroglobulinemia. Semin Oncol 2003;30:110-115.

2 Sahota SS, Forconi F, Ottensmeier CH et al. Origins of the malignant clone in typical Waldenstrom's macroglobulinemia. Semin Oncol 2003;30:136-141.

3 Leleu X, Jia X, Runnels J et al. The Akt pathway regulates survival and homing in Waldenstrom macroglobulinemia. Blood 2007;110:4417-4426.

4 Leleu X, Eeckhoute J, Jia X et al. Targeting NF-kappaB in Waldenstrom macroglobulinemia. Blood 2008;111: 5068-5077.

5 Roccaro AM, Sacco A, Husu EN et al. Dual targeting of the PI3K/Akt/mTOR pathway as an antitumor strategy in Waldenstrom macroglobulinemia.Blood 2010;115: 559-569.

6 Elsawa SF, Novak AJ, Ziesmer SC et al. Comprehensive analysis of tumor microenvironment cytokines in Waldenstrom macroglobulinemia identifies CCL5 as a novel modulator of IL-6 activity. Blood 2011;118: 5540-5549.

7 Hodge LS, Ziesmer SC, Yang ZZ et al. IL-21 in the bone marrow microenvironment contributes to IgM secretion and proliferation of malignant cells in Waldenstrom macroglobulinemia. Blood 2012;120:3774-3782.

8 Treon SP, Xu L, Yang G et al. MYD88 L265P somatic mutation in Waldenström's macroglobulinemia. N Engl J Med 2012;367:826-833.
9 Hunter ZR, Xu L, Yang G et al. The genomic landscape of Waldenstrom macroglobulinemia is characterized by highly recurring MYD88 and WHIM-like CXCR4 mutations, and small somatic deletions associated with B-cell lymphomagenesis. Blood 2014;123: 1637-1646.

10 Teitell MA. The TCL1 family of oncoproteins: coactivators of transformation. Nat Rev Cancer 2005;5: 640-648.

11 Herling M, Patel KA, Khalili J et al. TCL1 shows a regulated expression pattern in chronic lymphocytic leukemia that correlates with molecular subtypes and proliferative state. Leukemia 2006;20:280-285.

12 Herling M, Patel KA, Weit N et al. High TCL1 levels are a marker of B-cell receptor pathway responsiveness and adverse outcome in chronic lymphocytic leukemia. Blood 2009;114:4675-4686.

13 Bichi R, Shinton SA, Martin ES et al. Human chronic lymphocytic leukemia modeled in mouse by targeted TCL1 expression. Proc Natl Acad Sci USA 2002;99: 6955-6960.

14 Laine J, Künstle G, Obata $\mathrm{T}$ et al. The protooncogene TCL1 is an Akt kinase coactivator. Mol Cell 2000;6: 395-407.

15 Gaudio E, Spizzo R, Paduano F et al. Tcl1 interacts with Atm and enhances NF- $\mathrm{KB}$ activation in hematologic malignancies. Blood 2012;119:180-187.

16 Pekarsky Y, Palamarchuk A, Maximov V et al. Tcl1 functions as a transcriptional regulator and is directly involved in the pathogenesis of CLL. Proc Natl Acad Sci USA 2008;105:19643-8.

17 Herling M, Patel KA, Hsi ED et al. TCL1 in B-cell tumors retains its normal b-cell pattern of regulation and is a marker of differentiation stage. Am J Surg Pathol 2007;31:1123-1129.

18 Aggarwal M, Villuendas R, Gomez G et al. TCL1A expression delineates biological and clinical variability in B-cell lymphoma. Mod Pathol 2009;22:206-215.

19 QIAGEN Redwood City. IPA. Available on: http:// www.qiagen.com/ingenuity.

20 Swerdlow SH, Berger F, Pileri SA et al. Lymphoplasmacytic lymphoma. In: Swerdlow SH, Campo E, Harris NL et al. (eds). WHO Classification of Tumours of Haematopoietic and Lymphoid Tissues. 4th edn. Lyon, France: WHO/IARC: Lyon, France. 2008, pp 194-195.

21 Owen RG, Kyle RA, Stone MJ et al. Response assessment in Waldenström macroglobulinaemia: update from the VIth International Workshop. Br J Haematol 2013;160:171-176.

22 Rourke M, Anderson KC, Ghobrial IM. Review of clinical trials conducted in Waldenstrom macroglobulinemia and recommendations for reporting clinical trial responses in these patients. Leuk Lymphoma 2010;51: 1779-1792.

$23 \mathrm{R}$ Core Team. R: a language and environment for statistical computing [Internet]. $\mathrm{R}$ Foundation for Statistical Computing: Vienna, Austria; 2014. Available on: http://www.R-project.org.

24 Narducci MG, Pescarmona E, Lazzeri C et al. Regulation of TCL1 expression in B- and T-cell lymphomas and reactive lymphoid tissues. Cancer Res 2000;60: 2095-2100.

Supplementary Information accompanies the paper on Modern Pathology website (http://www.nature.com/ modpathol) 